\title{
ATLAS-BASED SEGMENTATION OF PATHOLOGICAL BRAIN MR IMAGES
}

\author{
M. Bach Cuadra, C. Pollo*, A. Bardera, O. Cuisenaire, J.-G. Villemure* and J.-Ph. Thiran \\ Swiss Federal Institute of Technology (EPFL), Signal Processing Institute (ITS) \\ $\mathrm{CH}-1015$ Lausanne, Switzerland \\ http://ltswww.epfl.ch/ brain \\ \{Meritxell.Bach,Olivier.Cuisenaire,JP.Thiran\}@epfl.ch \\ *Department of Neurosurgery \\ Lausanne University Hospital (CHUV), CH-1011 Lausanne, Switzerland
}

\begin{abstract}
We propose a method for brain atlas deformation in presence of large space-occupying tumors, based on an a priori model of lesion growth that assumes radial expansion of the lesion from its starting point. First, an affine registration brings the atlas and the patient into global correspondence. Then, the seeding of a synthetic tumor into the brain atlas provides a template for the lesion. Finally, the seeded atlas is deformed, combining a method derived from optical flow principles and a model of lesion growth (MLG). Results show that the method can be applied to the automatic segmentation of structures and substructures in brains with gross deformation, with important medical applications in neurosurgery, radiosurgery and radiotherapy.
\end{abstract}

\section{INTRODUCTION}

The use of deformable models to segment and project structures from a brain atlas onto a patient's Magnetic Resonance (MR) image is a widely used technique. Potential applications include segmentation of structures and substructures of the patient's brain for radiation therapy and presurgical planning. But, when large space-occupying tumors or lesions drastically alter shape and position of brain structures and substructures, atlas-based methods have been of limited use.

To the best of our knowledge, only two approaches related to atlas-based segmentation on pathological brains have been published. Kyriacou et al [1] propose to use a biomechanical model of the brain using finite-elements. The soft tissue deformations induced by the tumor growth are modelled first. Then they proceed to the registration with an anatomical atlas. Dawant et al [2] rely on a simpler approach using an optical-flow based technique instead of a complex model of biomechanics. The solution they propose is seeded atlas deformation (SAD), i.e., put a little seed with the same intensity properties as the lesion and then apply a non-rigid registration algorithm. But their approach usually involves the placement of a large seed that masks atlas structures leading in wrong results. The approach we present here is the continuation of the work presented in [3]. Our method instead of relying on the deformation calculation of the non-linear registration algorithm on the whole image, we apply an a priori model of tumor growth inside the tumor area, which assumes that the tumor has grown from a little seed in a radial fashion. As will be shown, this model allows the placing of a one voxel seed into the brain atlas and, therefore, minimizes the amount of atlas information that is masked by the tumor seed. We present results obtained on real patient images together with the assessment by an expert. The text is organized as follows. First, a brief description of the methods is done. Then, some results are presented followed by the discussion and conclusion.

\section{METHODS}

We propose a method for brain atlas deformation in presence of large space-occupying tumors, based on an a priori model of lesion growth that assumes radial expansion of the lesion from a seed voxel. For simplicity, only pushing lesions such as meningioma are considered. Hence, this method does not apply to infiltrating tumors or take into account the presence of the edema. The method works as follows. First, an affine transformation is applied to the brain atlas in order to globally match the patient's image. Also, the lesion is automatically segmented from the MRI. After that, the atlas is manually seeded with a voxel synthetic lesion placed on the estimated origin of the patient's lesion. Finally, the non-linear registration algorithm is performed in order to deform the seeded atlas to match the patient. It is applied only to the area outside the tumor location. Inside the tumor volume the a priori model of tumor growth is 
used. Finally, structures and substructures from the warped brain atlas may be projected onto the patient's image.

\subsection{Non-rigid deformation algorithm}

Relying on our previous experience [4], we use the demons algorithm proposed by Thirion [5]. This method approaches the problem of image matching as a diffusion process, in which object boundaries in the reference image are viewed as semi-permeable membranes. The floating image is considered as a deformable grid, and diffuses through these interfaces driven by the action of effectors (also called demons by analogy with Maxwell's demons) situated within the membranes. Various kinds of demons can be designed to apply this paradigm to specific applications. In the case of voxelby-voxel intensity similarity, the demons paradigm is similar to optical flow methods. In this study, the displacement vector for each voxel is:

$$
\vec{v}_{I_{2} \rightarrow I_{1}}=\frac{\left(I_{2}-I_{1}\right) \vec{\nabla} I_{1}}{\vec{\nabla} I_{1}^{2}+\left(I_{2}-I_{1}\right)^{2}},
$$

where $I_{1}$ and $I_{2}$ are the voxel intensities. In this approach, global smoothness of the displacement field is not enforced. Instead local constraint imposing similar displacements for nearby voxels are imposed by smoothing this field with a Gaussian filter. The choice of the smoothing parameter of the filter $\sigma$ is a key issue that has been previously studied in [6] and that will be also discussed in section 2.5.

\subsection{Affine transformation}

Before performing the non-rigid deformation algorithm, it is necessary to bring the atlas and patient volumes into global correspondence. Indeed the non-rigid registration technique needs overlapping of the patient and atlas structures in order to being able to match them. We apply an affine transformation to the brain atlas as proposed by Cuisenaire et al. [7].The optimal transformation looks for the coefficients that minimizes the Euclidian distance between the atlas cortical surface to the correspondent cortical surface in the target image.

\subsection{Lesion segmentation}

In order to apply the deformation method, a segmentation of the patient's lesion is needed. This segmentation is used first for the generation of the synthetic lesion seed and, second, for the construction of the model of tumor growth. The automated segmentation algorithm that has been used in this study is the Adaptive Template Moderated Spatially Varying Statistical Classification (ATM SVC) algorithm proposed by Warfield et al.[8]. The ATM SVC algorithm overcomes the limitations of spectral segmentation techniques and deformable models segmentation techniques by embedding a traditional k-Nearest Neighbors ( $\mathrm{k}-\mathrm{NN}$ ) classification into a higher dimensionality problem space. The additional dimensionality is derived from a brain atlas, and acts to moderate the statistical classification.

\subsection{Atlas seeding}

After the affine transformation, the atlas and the patient volumes are globally in correspondence except in regions that have been drastically deformed by the tumor. Atlas seeding consists of manually selecting the origin of the tumor growth in the healthy brain atlas. Unfortunately, when the seed is introduced, a masking of the information is done. Therefore, the optimum will be a seed of one voxel and all the voxels inside the lesion region should converge to it. Using the algorithm introduced in [2], this objective is impossible since an optical-flow based algorithm is used and large morphological differences can not be matched. In [3] we have introduced a preliminary model of lesion growth based on the gradient of the distance to the seed and a considerable reduction of the seed size was obtained. However, the seed was still bigger than one voxel and the seed position was fixed to the center of the lesion which is actually not medically realistic. In this paper, a radial growing of the seed to the edges until lesion edges is supposed, and it could be placed anywhere inside the lesion area.

\subsection{Non-rigid deformation using a model of tumor growth}

At this point, there is a template of lesion in the brain atlas, and there is an overlap between it and the patient's lesion. The elastic demons deformation is used outside the lesion. Inside, we assume a radial growth of the tumor from the tumor seed, according to the following equation:

$$
\vec{v}_{I_{2} \rightarrow I_{1} \text { lesion }}=\frac{\vec{D}_{\text {seed }}}{N_{\text {it }}},
$$

where $\vec{D}_{\text {seed }}$ is a vector that comes from the transformed point to the seed, $N_{i t}$ is the number of iterations of the deformation algorithm that have to be performed. With this transformation all the points inside the lesion area converge exactly to the seed voxel. Then, the entire field is regularized by the adaptative Gaussian filter to avoid possible discontinuities. Three zones of different elasticity are delimited: inside the lesion area the vector field induced by Eq. 2 is highly regular, and no smoothing is needed, i.e., $\sigma=0$. In the region close to the tumor we have large deformation due to the tumor growth, so it is necessary allow large elasticity, i.e., $\sigma$ should have a small value, typically $0.5 \mathrm{~mm}$. In the rest of the brain, deformations are smaller, due primarily to inter-patient anatomical variability. So larger $\sigma$ would be a better value, because it simulates a more rigid transformation. Previous studies [6], suggest a typical sigma to match 


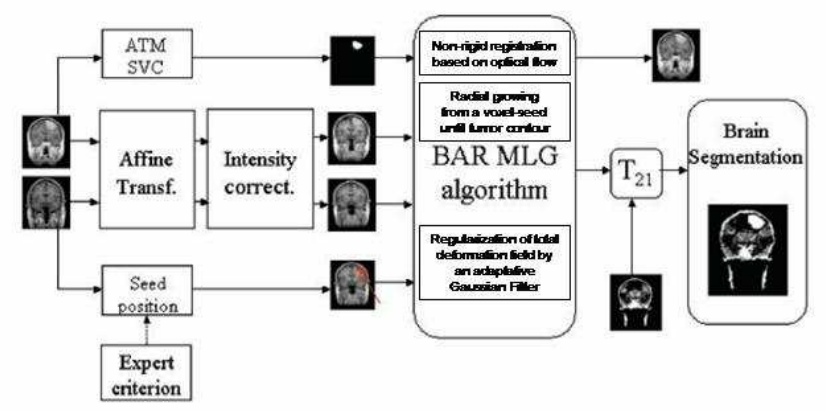

Fig. 1. Block diagram of the BAR MLG algorithm.

two healthy brains is about $0.8 \mathrm{~mm}$ and $1 \mathrm{~mm}$. In our case, a $\sigma=0.8$ is used. By proceeding in this way, the growth of the seed is tracked and the deformation force is adapted to the variations of this growth.

\section{RESULTS}

\subsection{Data sets}

The patient images have been retrieved from the Surgical Planning Laboratory (SPL) of the Harvard Medical School $\&$ NSG Brain Tumor Database ${ }^{1}$. They consist in volumes of 128 coronal slices of $256 \times 256$ pixels and $0.9375 \times$ $0.9375 \times 1.5 \mathrm{~mm}^{3}$ of voxel size and all of them have a meningioma. The digital atlas used in this work also comes from the SPL [9]. It is made of MR data from a single normal subject scanned with high resolution $256 \times 256 \times$ 160 volume data set in coronal orientation with $0.9375 \times$ $0.9375 \times 1.5$ voxel size. We have applied atlas-based registration using the model of lesion growth (BAR MLG) as presented in Fig. 1 and using the parameters defined in sections 2.3 and 2.5 .

\subsection{Deformed atlas images and deformation field}

In this section we compare our model to SAD from the point of view of the deformation field. Because of limited space, we present here this study only for one patient. This patient presents a left parasellar meningioma of approximately dimensions $41 \times 42 \times 52 \mathrm{~mm}^{3}$. We have performed $S A D$ for two different seed sizes (resulting from the tumor mask erosion of $8 \mathrm{~mm}$ and $12 \mathrm{~mm}$ respectively, see Fig. 2(a) and (b)), and the $M L G$ for one voxel seed (see Fig. 2(c)). With the largest seed, SAD achieves, in terms of deformed atlas images and deformation field, results that are comparable to those of our method (compare Fig. 2(d) and Fig. 2(f)). Note that the deformation field is almost the same for both methods, see Fig. 2(g) and 2(i)). On the contrary, when using the small seed, the deformation obtained by SAD method inside the tumor area does not reach the target (Fig. 2(e)). With SAD, the force on the lesion contour is actually misguided as we can see in Fig. 2(h). The different behavior between the two approaches can be explained as follows. While SAD relies on the intensity gradient for the deformation inside the tumor area, the MLG uses a model that applies the deformation independently from the intensity gradient and using only a priori information (i.e. a model of lesion growth). In the deformation field obtained by SAD, there is a strong gradient on the tumor and seed contour due to the highlighting. But between them, only the atlas gradient is used to lead the direction of the deformation inside the tumor. This gradient information is not enough when using a small seed since a large deformation is needed. That explains the dependency of SAD on the seed size and number of iterations. On the contrary, MLG can compensate these large differences thanks to the growing model.

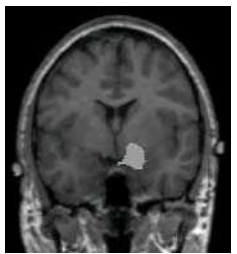

(a)

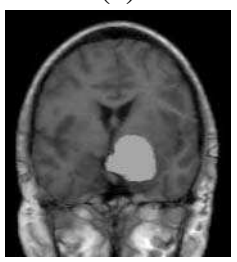

(d)

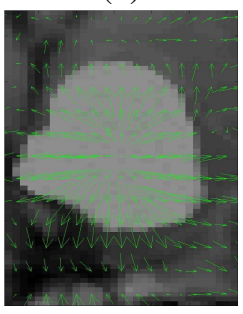

(g)

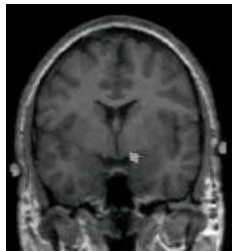

(b)

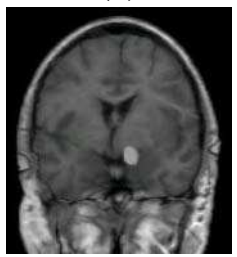

(e)

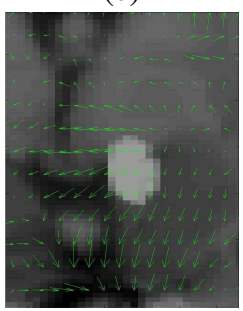

(h)

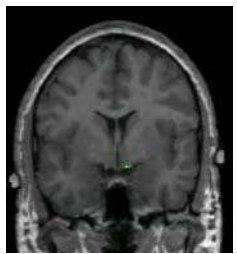

(c)

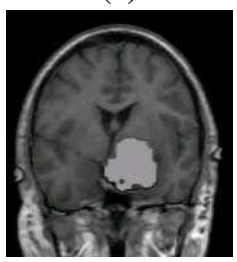

(f)

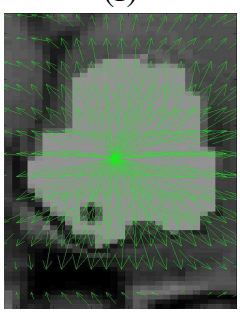

(i)
Fig. 2. Atlas seeding, lesion growth and deformation field analysis. (a) Warped atlas, big seed. (b) Warped atlas, small seed. (c) Warped atlas, one voxel seed (in green). (d) Deformation of seeded atlas with the big seed using SAD. (e) Deformation of seeded atlas with the small seed using SAD. (f) Deformation of seeded atlas with one voxel seed using MTG. (g) SAD: deformation fiel using a big seed. (h) SAD: deformation field using a small seed. (i) MTG: deformation field. NOTE: Deformation field corresponds to a zoom of the lesion.

${ }^{1}$ http://spl.bwh.harvard.edu:8000/ warfield/tumorbase/tumorbase.html 


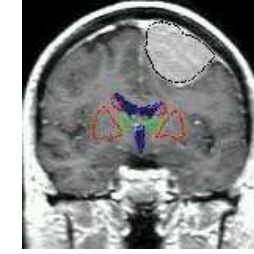

(a) Patient 1

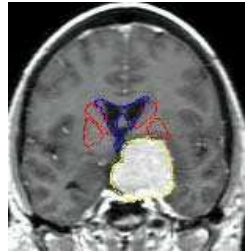

(b) Patient 2

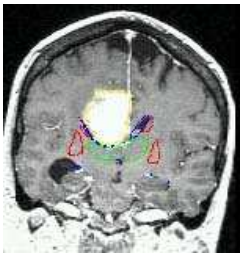

(c) Patient 3
Fig. 3. Results after applying the $B A R M L G$ algorithm.

\subsection{Segmentation results study}

In Fig. 3, structures and substructures from the deformed brain atlas have been projected to the patient's image. For 3 patients of the data set qualitative visual assessment of those structures by an expert was used for validation. The algorithm leads to good results for the 3 patients. Structures have been pushed to the correct place outside the tumor and well deformed. In a near future, we will also present some quantitative measures comparing the overlap between automatic and manual segmentations.

\section{DISCUSSION}

Our work presents three main differences respect to the most similar approach in the literature [2]. First, automated segmentation of the patient's lesion is performed instead of manually drawing the tumor contour. Second, we apply an a priori model of tumor growth inside the lesion area, which assumes that the tumor has grown in a radial way. This model of lesion growth is even much simpler as the one presented before in [3], because no gradient of the distance map between the seed and the tumor mask has to be calculated. Then, less computational time and memory resources are needed. Also, by using the model of lesion growth, dependence on the number of iterations of the non-rigid deformation algorithm is eliminated inside the tumor area (see Eq. 2). Third, deformation is applied only once while in [2] the demons algorithm is applied twice: first to match the non large deformed structures and then to match the structures close to the lesion, highly deformed. Notice that is not exactly the same to perform twice a non-rigid registration technique (first rigidly and then more elastically) than perform only once. It can be proved that successive applications of a non-rigid registration algorithm as the optical flow can result more in a fluid than an elastic registration [10]. However, there is a drawback to these improvements: the seed position, which simulates where the tumor has begun to grow, has to be manually chosen by an expert. This is a hard task which usually requires some tries before we get the correct initial position. Notice that the algorithm is quite sensitive to this position since the structures can be pushed completely wrong if the starting point is not realistic.

\section{CONCLUSION}

We proposed a new approach for brain atlas deformation in the presence of large space-occupying tumors, which makes use of a simple model of tumor growth. The use of an $a$ priori model for the brain atlas deformation inside the tumor area enables a good matching, even when brain structures have been drastically altered by the presence of a tumor. Results show that our method overcomes the limitation such as the seed size dependence and convergence to the target that the most similar article in the literature had.

\section{ACKNOWLEDGEMENT}

We wish to thank Dr Ron Kikinis who has provided us with the Surgical Planning Laboratory (SPL) atlas. Also we would like to thank NSG Brain Tumor Database who has provided us with patient images. This work is supported by the Swiss National Science Foundation grants numbers 21-55580-98 and 20-64947.01.

\section{REFERENCES}

[1] S.K. Kyriacou and C. Davatzikos, "Nonlinear elastic registration of brain images with tumor pathology using a biomechanical model," IEEE Trans. Med. Img, vol. 18, no. 7, pp. 580-592, 1999.

[2] B.M. Dawant et al., "Brain Atlas Deformation in the Presence of Large Space-occupying Tumors," in MICCAI, 1999, pp. 589-596.

[3] M. Bach Cuadra et al., "Atlas-based Segmentation of Pathological Brains usiing a Model of Tumor Growth," in $M I C$ CAI, 2002, pp. 380-387.

[4] M. Bach Cuadra et al., "Automatic segmentation of internal structures of the brain in MRI using a tandem of affine and non-rigid registration of an anatomical atlas," in ICIP, October 2001.

[5] J.P. Thirion, "Fast Non-Rigid Matching of 3D Medical Images,” Tech. Rep. 2547, INRIA, May 1995.

[6] M. Bach et al., "Atlas-based Segmentation of Pathological Brains using a Model of Tumor Growth," Tech. Rep., ITSEPFL, February 2002.

[7] O. Cuisenaire et al., "Automatic Registration of 3D MR images with a Computerized Brain Atlas," in SPIE Med. Img., 1996, vol. 1719, pp. 438-449.

[8] S.K. Warfield et al., "Adaptive, Template Moderated, Spatially Varying Statistical Classification," Med. Img. Analy., vol. 4, no. 1, pp. 43-55, March 2000.

[9] R. Kikinis et al., "A digital brain atlas for surgical planning, model driven segmentation and teaching," IEEE Trans. on Vis. and Comp. Graph., vol. 2, no. 3, 1996.

[10] P. Cachier et al., "Fast Non-Rigid Matching by Gradient Descent: Study and Improvements of the "Demons" Algorithm," Tech. Rep. 3706, INRIA, June 1999. 